\title{
Management of Women with Vaginismus-related Infertility Through Assisted Reproductive Techniques
}

\author{
(1) Arzu Yurci1, (1) Şaban Karayağız² \\ ${ }^{1}$ Memorial Kayseri In Vitro Fertilization Center, Department of Obstetrics and Gynecology, Kayseri, Turkey \\ ${ }^{2}$ Nuh Naci Yazgan University Faculty of Science, Department of Psychology, Kayseri, Turkey
}

Cite this article as: Yurci A, Karayağız Ş. Management of Women with Vaginismus-related Infertility Through Assisted Reproductive Techniques. J Acad Res Med 2021;11(2):174-8

\begin{abstract}
Objective: To investigate reproductive outcomes of patients with infertility due to severe vaginismus undergoing in vitro fertilisation and embryo transfer (IVF-ET).

Methods: Twenty-nine patients were selected among patients who presented with complaints of vaginismus-related infertility. The diagnosis and severity of vaginismus were assessed according to the Lamont-Pacik system. Five women with milder forms of vaginismus were treated with other options. Of the remaining 24 patients with severe vaginismus, 20 had Lamont grade 3 and four with Lamont grade 4. Likewise, 24 patients who were diagnosed with unexplained infertility were selected as the control group.

Results: Of the 24 patients, 15 had first attempt, 8 had second and 1 had fourth attempt. Folliculometry was performed with abdominal ultrasonography in 21 women with normal body mass index. Folliculometry and endometrial evaluation were performed in three overweight cases with transvaginal ultrasonography under sedation. Single ET was performed in $27 \mathrm{cycles}$, and two ETs were performed in the others. Beta human chorionic gonadotropin positivity was detected in 20 of the 39 cycles. Similarly, in 20 patients, while clinical pregnancy was detected (20 of 39 cycles, $51.2 \%)$, the number of cycles with term and live births were 16 (16 of 39 cycles, $41.0 \%$ ). While clinical pregnancy was detected in 25 of 35 cycles in the control group (71.4\%), live birth was achieved in 22 cycles (62.8\%). Compared with the vaginismus group, clinical pregnancy rate $(20 \mathrm{vs} 25, \mathrm{p}<0.09)$ and live birth rate $(\mathrm{LBR})(16$ vs $22, \mathrm{p}<0.06)$ were comparable.

Conclusion: Both clinical pregnancy and LBRs of women with infertility due to severe vaginismus undergoing IVF-ET are similar to women with unexplained infertility.
\end{abstract}

Keywords: Vaginismus, Lamont-Pacik scale, IVF-ET, fertility outcome

\section{INTRODUCTION}

Vaginismus is the recurrent and involuntary contraction of the paravaginal muscles, which interferes with sexual intercourse and may cause sexual penetration even impossible $(1,2)$. It has both psychological and physical components and meets the fourth edition of Diagnostic and Statistical Manual of Mental Disorders Text Revision criteria $(1,3)$. The definition of vaginismus was recently modified, and the fifth edition of the Diagnostic and
Statistical Manual of Mental Disorders defined vaginismus as a subset of 'genito-pelvic pain and penetration disorder' (4). The diagnosis of vaginismus is made by detailed history and physical examination. The prevalence of vaginismus varies between $5 \%$ and $7 \%$, and it is one of the more prevalent female sexual dysfunctions (5). It is divided into primary and secondary vaginismus. Primary vaginismus occurs when the woman has never been able to have penetrative intercourse. Secondary vaginismus occurs when a

ORCID IDs of the authors: A.Y. 0000-0003-4808-9019; Ş.K. 0000-0002-6914-4630. 
woman has previously been able to have intercourse but cannot be penetrated anymore. Vaginismus can be mild, in which different treatments and sexual intercourse are possible; or severe, making treatment or sexual intercourse impossible (6). Since these patients cannot have sexual intercourse, it is not possible to have a natural pregnancy.

As with other sexual dysfunctions, vaginismus can lead to marital problems (7) and likely to result in infertility. To achieve pregnancy in women with vaginismus, it is necessary to either perform intrauterine insemination (IUI) under sedation or to direct the patient to assisted reproductive techniques (ART). However, in cases where both IUI and in vitro fertilisation and embryo transfer (IVF-ET) are planned, performing folliculometry appears to be an important problem. In severe vaginismus, the vaginal muscles respond to any stimulus with increased contraction, and these patients cannot be evaluated by gynaecological examination or transvaginal ultrasonography (USG) without sedation (6). Abdominal USG may allow folliculometry and endometrial evaluation in cases with normal body mass index (BMI), but folliculometry with abdominal USG may not provide very clear information in obese cases. Women with vaginismus who cannot tolerate a transvaginal examination might have an examination under anaesthesia during which any vaginal spasm disappears. For all these reasons, patients with vaginismus who take ovulation stimulation drugs for IUI or IVF-ET may need to undergo a sedation anaesthesia two or three times during folliculometry.

Classifying patients with vaginismus according to the severity of the disease before taking IUI or IVF-ET treatment may make it possible to help patients further during the treatment. For this reason, we classified all vaginismus cases according to LamontPacik system and then introduced them into their corresponding treatment $(6,8)$. This scale classifies patients according to their response to the pelvic examination and penetration history. In this study, IVF-ET results of women diagnosed with severe vaginismus are presented in detail. To the best of our knowledge, this is the largest series on ART protocols and fertility outcomes of women with infertility due to severe vaginismus.

\section{METHODS}

In this retrospective case-control study, study participants were selected among patients who presented to our clinic with complaints of infertility due to vaginismus. Medical data were collected from our patient database. A total of 29 patients ( 3 had secondary infertility, 26 had primary infertility) who were admitted to the Memorial Kayseri Hospital IVF Centre between December 2012 and June 2020 were included in the study. Patients with other underlying infertility problems such as decreased ovarian reserve, male factor, endometriosis/endometrioma and polycystic ovary syndrome were excluded from the study. Moreover, 24 patients who were diagnosed with unexplained infertility and for whom IVF-ET decision was made were selected as the control group.

This study was conducted in accordance with the Declaration of Helsinki. Ethical approval was obtained from the Ethics
Committee of the Nuh Naci Yazgan University (decision no: 2020/2, date: 20.08.2020), and written informed consent was obtained from all participants at the time of enrolment. While some of the patients already presented with vaginismus, some were diagnosed with vaginismus following examination. In patients who presented with vaginismus, initial pelvic examination was performed without sedation, and a diagnosis of vaginismus was confirmed. The severity of vaginismus was assessed according to the Lamont scale. This scale was based on the patient's history, their behaviour during a routine gynaecologic examination, and physical demonstration of perineal muscular spasm $(6,8)$. While three cases were Lamont grade 1 (first-degree vaginismus), two cases were Lamont grade 2 (second-degree vaginismus). These five women with milder forms of vaginismus cooperated with different treatment suggestions such as vaginal dilators, biofeedback therapy, psychotherapy, hypnotherapy and lubricant treatments. A total of 20 patients with Lamont grade 3 (third-degree vaginismus) and four with Lamont grade 4 (fourth-degree vaginismus) were diagnosed with serious vaginismus and were included in the study (Table 1). Of the 24 patients, three were previously pregnant with IUI, but they were included in the study as having secondary infertility because they could not conceive again. The remaining 21 patients were accepted as having primary infertility, who were terrified of any attempted vaginal penetration and declined treatment suggestions. As a result of the interviews with the patients, eight patients proceeded with IUI, while 13 patients who declined the IUI recommendation were taken directly to the IVF programme. Failure of two IUIs was declared as IUI failure. Pregnancy could not be obtained in any of the eight patients with at least two IUIs, and they were directed to IVFET. As a result, all 24 female patients ( 21 with primary infertility and three with secondary infertility) diagnosed with severe vaginismus were included in the IVF-ET programme. Antral follicle count and endometrial evaluation were performed with abdominal USG carried out on day 3 of the menstrual cycle. Likewise, on day 3 of the spontaneous cycle, basal hormonal levels of patients in the vaginismus and control groups were measured. A hysterosalpingogram was performed under sedation in all cases, and information was obtained about the fallopian tubes and endometrial cavity. Propofol $(2 \mathrm{mg} / \mathrm{kg}$, Polifarma, Tekirdağ, Turkey) was administered intravenously during sedation anaesthesia.

Patients were asked for psychiatric consultation before initiating controlled ovarian stimulation, and then detailed information about the treatment process was given. They were informed about follicle follow-up with abdominal USG, but folliculometry could be performed with USG under sedation if necessary, and their consent was obtained. All women with severe vaginismus were treated according to a standard antagonist protocol with individually dosed recombinant follicle-stimulating hormone starting on days 2-3 of the menstrual cycle. Gonadotrophinreleasing hormone antagonist was started on day 5 or 6 of 
stimulation. When at least three follicles reached $16-17 \mathrm{~mm}$ in diameter, maturation of follicles was induced with recombinant human chorionic gonadotropin (hCG) (Ovitrelle, Merck-Serono, 250 mg, Modugno, BA, Italy). In 21 of 24 cases, abdominal USG provided sufficient images of both follicle sizes and endometrial thickness, and no sedation was required. In the remaining three patients who were obese, folliculometry was performed under sedation each time, since follicle sizes were not clearly evaluated in abdominal USG. In all cases, serum oestradiol levels were measured at least three times during follow-up to minimise follicle measurement errors due to abdominal USG. Owing to the highresolution quality of the USG used for folliculometry (GE Voluson 730), it was possible to follow the follicles in a healthy way, and the hCG application day was determined correctly. Oocyte collection was performed $36 \mathrm{~h}$ after hCG application. Ovarian follicles were aspirated using a single-lumen, 17-gauge needle (Cook Medical, Bloomington, IN, USA) guided by transvaginal USG.

\section{Statistical Analysis}

Statistical analyses were performed with the SPSS 20.0 software. Data were shown as mean \pm standard deviation values or percentage. Percentages of demographic findings were compared using the paired t-test. A $p<0.05$ was considered significant.

\section{RESULTS}

Demographic characteristics of the vaginismus and control groups are shown in Table 2. This study included 24 patients with severe vaginismus, and the total number of IVF attempt performed on these patients was 35. Likewise, 35 cycles were performed on 24 patients in the control group. While 15 of 24 patients had their first attempt, eight patients had their second, and one patient had their fourth. Of the 35 cycles, 28 were fresh cycles and seven were freeze-thaw cycles. These three patients with infertility achieved their first pregnancy with IUI. However, they were directed to IVF because they could not get results from their IUI (transfer of washed sperm into the endometrium) trials for the second pregnancy. Of the 21 patients with primary infertility, eight tried IUI, but they were directed to IVF because pregnancy could not be obtained. The remaining 13 patients proceeded directly with IVF because they declined IUI. Folliculometry (measurement of follicle count and size) and endometrial evaluation was performed in three patients with $\mathrm{BMI}>30$ with transvaginal scanning and under sedation. BMI of these patients were recorded as 34, 32 and 37 , respectively. By contrast, in 21 patients with BMI within

\section{Table 1. Classification of 24 women with vaginismus according to the Lamont-Pacik scale}

\begin{tabular}{|c|c|c|c|}
\hline Lamont grade & Severity & The number of patients (\%) & IVF-ET \\
\hline Lamont grade 1 & First-degree vaginismus & $3(10.3 \%)$ & 3 (follow-up) \\
\hline Lamont grade 2 & Second-degree vaginismus & $2(6.8 \%)$ & 2 (follow-up) \\
\hline Lamont grade 4 & Fourth-degree vaginismus* & $4(13.7 \%)$ & 4 (IVF-ET) \\
\hline
\end{tabular}

Table 2. Demographic characteristics of participants with infertility due to vaginismus and control participants

\begin{tabular}{|c|c|c|c|}
\hline Characteristics & $\begin{array}{l}\text { Vaginismus } \\
(n=24)\end{array}$ & $\begin{array}{l}\text { Control } \\
(n=24)\end{array}$ & $p$ \\
\hline Age (years) & $29.3 \pm 4.4$ & $29.0 \pm 3.7$ & 0.678 \\
\hline Infertility duration (years) & $4.9 \pm 2.9$ & $6.0 \pm 3.3$ & 0.129 \\
\hline IVF attempt, n & $1.5 \pm 0.9$ & $1.6 \pm 0.8$ & 0.611 \\
\hline Day 2 E2 (pg/mL) & $26.1 \pm 8.0$ & $27.3 \pm 7.3$ & 0.475 \\
\hline E2 on hCG day (pg/mL) & $1338.6 \pm 453$ & $1148.3 \pm 486$ & 0.127 \\
\hline Day 2 P4 (ng/mL) & $0.28 \pm 0.1$ & $0.25 \pm 0.1$ & 0.183 \\
\hline P4 on hCG day (ng/mL) & $0.79 \pm 0.3$ & $0.81 \pm 0.3$ & 0.781 \\
\hline Total oocyte, n (per cycle) & $11.2 \pm 4.9$ & $9.94 \pm 3.8$ & 0.277 \\
\hline Mll oocyte, n & $8.71 \pm 3.5$ & $7.58 \pm 3.1$ & 0.192 \\
\hline 2 PN, n (per cycle) & $6.61 \pm 3.0$ & $6.00 \pm 2.7$ & 0.413 \\
\hline Fresh or thaw cycle, $n$ & $31 \mathrm{fresh} / 8$ thaw & $28 \mathrm{fresh} / 7$ thaw & 1.0 \\
\hline Endometrial thickness (mm) & $9.4 \pm 1.1$ & $9.7 \pm 1.4$ & 0.401 \\
\hline $\mathrm{CPR}^{\star}, \mathrm{n}$ & 20 of 39 cycles (51.2\%) & 25 of 35 cycles $(71.4 \%)$ & 0.097 \\
\hline $\mathrm{LBR}^{*}, \mathrm{n}$ & 16 of 39 cycles (41.0\%) & 22 of 35 cycles (62.8\%) & 0.068 \\
\hline
\end{tabular}


normal limits, folliculometry was performed with abdominal USG without sedation. Follicle measurements made with abdominal USG coincided with the measurements taken during the ovum pick-up (method for egg collection) in terms of both follicle size and number. ET (transfer of fertilised egg to the endometrial cavity via catheter) was performed on day 3 in 23 of the 35 cycles and day 5 in the remaining 12 cycles. Single ET was performed in a total of 27 cycles. In seven patients aged $>35$ years or were having their third or fourth attempt, two ETs were performed. Regardless of the infertility status, ET was performed under sedation in all cases.

Beta hCG positivity was detected in 20 of the 39 cycles. Similarly, in 20 cases, while clinical pregnancy was detected (20 of 39 cycles, $51.2 \%$ ), the number of cycles with live births were 16 (16 of 39 cycles, $41.0 \%$ ). While clinical pregnancy was detected in 25 of 35 cycles in the control group (71.4\%), live birth was achieved in 22 cycles (62.8\%). Compared with the vaginismus group, clinical pregnancy rate [clinical pregnancy rate $(C P R) ; 20$ vs $25, p<0.09$ ] and live birth rate (LBR) were comparable (16 vs. $22, p<0.06$ ). Although there was an increasing trend in CPR and LBR in the control group, the difference was not significant. No significant difference was found between the control group and vaginismus group in terms of other measured parameters (Table 2).

\section{DISCUSSION}

Vaginismus is a psychological and physical disorder manifested by fear and anxiety to penetration of the penis to vagina, and it is thought to be one of the most common female psychosexual dysfunctions (6). In the literature reviewed, no study was related to the management of infertile cases diagnosed with severe vaginismus, except case reports and small case series. Since it is not unusual to conduct a detailed research on women with vaginismus, it is not possible to describe clearly the fertility status of the patient and to determine the treatment method accordingly. In fact, it is incorrect to mention cases diagnosed as vaginismus as infertility. In mild vaginismus cases, different treatment approaches can be effective, whereas in severe cases, treatment is very difficult. For this reason, there is no option other than ART in patients who have been diagnosed with severe vaginismus and who desired pregnancy (9).

At present, many authors have regarded severe cases of vaginismus as the cause of infertility. Indeed, involuntary contractions in the paravaginal muscles sometimes lead to painful intercourse, sometimes making sexual intercourse impossible. By contrast, underlying psychosexual causes lead to loss of libido over time, contributing to the isolation of couples and thus to infertility $(9,10)$. To our knowledge, there is no comprehensive research on the management of patients with vaginismus having infertility complaints. Most studies have been in the form of case reports and have focused on the diagnosis and treatment of vaginismus. In these studies, since patients were not classified according to the severity of vaginismus, all cases with mild, moderate, and severe vaginismus were placed in the same pool and treated as having a single form of disease. However, vaginismus has various subforms, with both psychological and physical components. Lamont and Pacik classified vaginismus according to their severity and offered treatment accordingly $(6,8)$. Although a study suggested that patients with vaginismus can become pregnant with ejaculation exterior to the vagina, most patients require one of the ART techniques. Recently, Achour et al. (10) reported that 13 of 20 patients with vaginismus became pregnant with incomplete sexual intercourse without penetration. However, the authors did not specify the classification of vaginismus diagnosis and severity. This is a serious limitation, and data on vaginismus cases with spontaneous pregnancy are insufficient.

In the present study, we first confirmed the diagnosis of patients who presented with vaginismus complaints and who wanted to become pregnant. Then, we divided the patients into groups according to the Lamont scale $(6,8)$. After classification, five patients with mild and moderate vaginismus (Lamont grade 1 and 2) were directed to personalised vaginismus treatment. Of these five patients, three had sexual intercourse after the treatments. The remaining two patients did not benefit from treatments for vaginismus. Classification of the severity of vaginismus is important as it assists in determining whether the patient will be directed to natural cycle or IVF-ET. Mild vaginismus may respond to sexual intercourse or IUI, whereas severe vaginismus requires IVF-ET. In patients with infertility due to vaginismus, disease severity should be evaluated according to the Lamont or Pacik scale and a treatment plan should be drawn accordingly. In patients with severe vaginismus (third or fourth degree), these cases should be directed to IUI or IVF as it is impossible to perform pelvic examination or transvaginal USG. Approximately $69 \%$ of our patients who presented with infertility complaint had third-degree vaginismus and $13 \%$ had fourth-degree vaginismus. None of the eight patients who had IUI from both groups could conceive, so they were referred to IVF-ET. As a result, clinical pregnancy was detected in 20 of the patients who underwent IVF-ET because of third- or fourth-degree vaginismus (57\%), while term and live births were obtained in 16 cases (45.7\%). In the light of these data, we can argue that in severe vaginismus, IUI is also futile similar to vaginal dilators, physical therapy with or without biofeedback, sex and relationship counselling, psychotherapy, cognitive behavioural therapy, hypnotherapy and lubricant treatments. For this reason, patients with severe vaginismus should be directed to IVF-ET immediately, and the loss of ovarian reserve should be prevented by averting delay due to palliative treatments.

Stratifying the severity of vaginismus in infertile cases helps the clinician choose among numerous infertility treatment options to better understand the disease form and patient's capability. Compared with other infertility disorders such as tubal factor or male factor infertility, treatment of vaginismus has potential for a high success rate following IVF-ET. In this study, approximately half of the patients achieved term pregnancy with IVF-ET. However, in severe cases, IUI failed. However, explaining the reason for IUI failure was the most challenging point. Three patients with severe 
vaginismus had previously achieved pregnancy with IUI. However, they could not conceive in their subsequent IUI. We believe that failure of subsequent IUI trials, despite successful pregnancy in the first IUI, was related to the individual characteristics of the patients rather than the severity of vaginismus. During our study, women with severe vaginismus had greater fear and anxiety to pelvic examination and had more difficulty with infertility treatment recommendations than women with milder forms of vaginismus. By contrast, many cases demonstrated palpitations, hyperventilation, trembling, uncontrollable shaking, screaming, hysteria, wanting to jump off the table, nausea and vomiting, both before and during sedation. In addition to fear and anxiety, all these visceral reactions encountered during IUI or ET may cause undesirable contractions in the sub-endometrial area, leading to decreased pregnancy rates. However, this idea should be clarified with further studies.

\section{Study Limitations}

Although this study presents original data, it has some limitations. Despite the low number of cases, this is the most comprehensive study addressing the management of vaginismus with ART. The fact that vaginismus is not considered a major problem in Western societies and this disease appears to be more of a problem in Middle East and Middle Asian societies does not weaken the power of this study. Despite all these limitations, this study provides comprehensive tips on management of infertile vaginismus cases.

\section{CONCLUSION}

This study is the most comprehensive series of management of infertility due to severe vaginismus, despite the small number of cases. In this study, patients with vaginismus more often presented to infertility clinics. Rather than providing patients with vaginismus directly with infertility treatment, classifying them according to the disease severity and treating them accordingly made the results more successful. In addition, classifying cases helps in the selection of treatable cases and individualisation of treatment. In severe vaginismus, couples may be directed to IUI and then IVF-ET. The CPR and LBR of women with infertility due to severe vaginismus receiving IVF-ET treatment are close to the infertile population without vaginismus. For this reason, IVF-ET may be one of the possible treatment options in the management of patients with infertility presenting with severe vaginismus.

Ethics Committee Approval: Ethical approval was obtained from the Ethics Committee of the Nuh Naci Yazgan University (decision no: 2020/2, date: 20.08.2020).

Informed Consent: Written informed consent was obtained from all participants at the time of enrolment.

Peer-review: Externally peer-reviewed.

Author Contributions: Surgical and Medical Practices - A.Y.; Concept A.Y., Ş.K.; Design - A.Y.; Data Collection and/or Processing - A.Y., Ş.K.; Analysis and/or Interpretation - A.Y.; Literature Search - A.Y., Ş.K.; Writing - A.Y., S..K.

Conflict of Interest: The authors have no conflict of interest to declare.

Financial Disclosure: The authors declared that this study has received no financial support.

\section{REFERENCES}

1. Basson R, Althof S, Davis S, Fugl-Meyer K, Goldstein I, Leiblum S, et al. Summary of the recommendations on sexual dysfunctions in women. J Sex Med 2004; 1: 24-34.

2. Amidu N, Owiredu W, Woo E, Addai Mensah O, Quaye L, Alhassan A, et al. Incidence of sexual dysfunction: a prospective survey in Ghanaian females. Reprod Biol Endocrinol 2010; 8: 106-11.

3. Bravo CS, Melendez JC, Ramirez SM, Lopez ME. Feminine and masculine sexual dysfunctions: gender comparison in a Mexico City sample [Disfunciones sexuales femininas y masculinas: Comparacion de genero en una muestra de la Ciudad de Mexico]. Salud Mental 2005; 28: 74-80.

4. American Psychiatric Association. Diagnostic and Statistical Manual of Mental Disorders DSM-V, 5th ed. Washington, DC: American Psychiatric Association; 2013

5. Spector IP, Carey MP. Incidence and prevalence of the sexual dysfunctions: a critical review of the empirical literature. Arch Sex Behav 1990; 19: 389408.

6. Pacik PT. Understanding and treating vaginismus: a multimodal approach. Int Urogynecol J 2014; 25: 1613-20.

7. Catalan J, Bradley M, Gallwey J, Hawton K. Sexual dysfunction and psychiatric morbidity in patients attending a clinic for sexually transmitted diseases. Br J Psychiatry 1981; 138: 292-6.

8. Lamont JA. Vaginismus. Am J Obstet Gynecol 1978; 131: 633-66.

9. Souza MDCB, Gusmão MCG, Antunes RA, Souza MM, Rito ALS, Lira P, et al. Vaginismus in Assisted Reproductive Technology Centers: an invisible population in need of care. JBRA Assist Reprod 2018; 22: 35-41.

10. Achour R, Koch M, Zgueb Y, Ouali U, Ben Hmid R. Vaginismus and pregnancy: epidemiological profile and management difficulties. Psychol Res Behav Manag 2019; 12: 137-43. 